\title{
The Use of Hypertonic Saline and Hetastarch in an American Kestrel (Falco sparverius) with Traumatic Brain Injury
}

\author{
Anne E. Rivas, Julia K. Whittington, DVM, Jessica A. Emerson, DVM \\ Department of Veterinary Clinical Medicine \\ University of Illinois College of Veterinary Medicine \\ URBANA, ILLINOIS
}

\begin{abstract}
An adult male American kestrel was presented to the Wildlife Medical Clinic at the University of Illinois by a motorist that witnessed the bird collide with a car. On presentation the kestrel was quiet, alert, responsive, and able to fly. However, over the next two hours the bird became obtunded, laterally recumbent, and lacked a pupillary light reflex in the left eye. Intravenous catheterization was performed and a bolus of hypertonic saline with hetastarch was administered to combat suspected increased intracranial pressure. The bolus was repeated during the first 12 hours. Additional therapy included administration of an anti-inflammatory agent, subdued lighting with minimal stimulation, and oxygen therapy. After two days the bird began to perch, and by five days the left eye was assessed as visual again. By day seven, the patient was deemed recovered, and was subsequently transferred to a raptor rehabilitation facility to recondition prior to release. This case suggests the usefulness of hypertonic saline with hetastarch as a treatment for traumatic brain injury in a wildlife rehabilitation setting.
\end{abstract}

Key words: Traumatic brain injury, hypertonic saline, wildlife rehabilitation

\section{INTRODUCTION}

Many patients present to wildlife hospitals and rehabilitation facilities due to trauma. Of these trauma cases, patients with head trauma and traumatic brain injuries (TBI) can be some of the most frustrating and challenging to treat. Causes of head trauma are varied and can range from impact injuries, predatory attacks, or projectile injuries (e.g. gunshot wounds). Primary brain injury resulting in hemorrhage, contusions, and nerve cell death occurs at the time of the initial traumatic event and is seldom treatable. Secondary

Anne E. Rivas is a third year veterinary student at the University of Illinois College of Veterinary Medicine and student manger of the Wildlife Medical Clinic.

Julia K. Whittington, DVM is a clinical associate professor in the Department of Veterinary Clinical Medicine at the University of Illinois and serves as the medical director of the Wildlife Medical Clinic.

Corina Jessica A. Emerson, DVM was the zoological medicine intern at the University of Illinois Veterinary Teaching Hospital and is currently completing a zoological medicine residency at the University of Florida. brain injury refers to the progressive neuronal damage that follows the initial injury and is due to a variety of pathophysiological mechanisms. Treatment should be focused on limiting the damage caused by secondary brain injury (Syring 2005).

A key component of this treatment is management of cerebral edema and the associated increased intracranial pressure (ICP). Administration of hyperosmotic agents such as mannitol and hypertonic saline can help decrease ICP in these cases by increasing the osmolality of the blood and drawing fluid off the brain. Mannitol is currently the standard treatment for cases of increased ICP both in human and veterinary medicine (Bullock et al 2000). Hypertonic saline has been shown to be effective in reducing ICP and has some advantages over mannitol, including its ease of administration and accessibility. This paper describes the use of hypertonic saline with hetastarch as an effective treatment of suspected increased ICP in a wildlife patient.

\section{CASE REPORT}

A $0.11 \mathrm{~kg}$ adult male American kestrel (Falco sparverius) was presented to the Wildlife Medical Clinic at the University of Illinois by a motorist who witnessed the bird collide with a car. On presentation, the kestrel was quiet, alert, and responsive and was noted to have a body condition score of $3 / 5$ based on pectoral muscle evaluation. Mucus membranes were pink and moist, and the capillary refill time was less than two seconds. Fresh blood was observed in the oral cavity and on the tail feathers, with no other signs of trauma noted. The kestrel was witnessed to have normal flight capabilities after presentation. A $1.0 \mathrm{mg} / \mathrm{kg}$ dose of carprofen (Rimadyl, Pfizer, New York, NY) was administered by mouth to combat potential inflammation and pain associated with the trauma, and the bird was placed in a quiet cage. 
When observed two hours later, the kestrel was obtunded, laterally recumbent to the left, and lacked a pupillary light reflex in the left eye (OS). Mucus membranes had become dry and pale, and the patient had significantly decreased peripheral perfusion based on assessment of the basilic vein. A 24-gauge intravenous catheter was placed in the right medial metatarsal vein. Warmed hetastarch $(6 \%$ in $0.9 \%$ percent saline, Novaplus, Lake Forest, IL) at $3.0 \mathrm{ml} / \mathrm{kg}$ and hypertonic saline (7.2\%, Bimeda, Inc., Le Sueur, MN) at $3.0 \mathrm{ml} / \mathrm{kg}$ were bolused over 15 minutes to combat suspected elevation in ICP. The patient subsequently was started on intravenous Normosol ${ }^{\circledR}{ }_{-} \mathrm{R}(124 \mathrm{ml} /$ $\mathrm{kg} /$ day, Abbott Animal Health, Abbott, IL) to provide maintenance fluids and replace perceived fluid deficit. Additional therapy included subdued lighting with minimal stimulation, keeping the patient sternal with the head elevated at a $30^{\circ}$ angle, and oxygen therapy administered via an oxygen concentrator $(60 \%$ fraction of inspired oxygen).

The kestrel's condition slightly improved after the initial therapy as indicated by increased mental awareness. However, the hypertonic saline and hetastarch boluses were repeated within the first twelve hours when the kestrel continued to exhibit depressed mentation and remained laterally recumbent. In order to limit handling and its associated stress for the first 48 hours, non-steroidal anti-inflammatory therapy with meloxicam (Metacam ${ }^{\circledR}$, Boehringer Ingelheim Vetmedica, Inc., St. Joseph, MO) at a dose of $0.5 \mathrm{mg} /$ $\mathrm{kg}$ given intramuscularly once daily replaced the carprofen therapy. Intravenous fluids were administered to the kestrel to achieve and maintain appropriate hydration (Normosol ${ }^{\circledR}-\mathrm{R}, 60-80 \mathrm{ml} / \mathrm{kg} /$ day). After 48 hours of treatment, the bird began perching and was more responsive to stimuli but would return to lateral recumbency after moderate stress. He also did not track movement or demonstrate an OS pupillary light reflex. Oxygen therapy was discontinued and gavage feeding with Carnivore Care slurry (Oxbow Animal Health, Murdock, NE) was begun. Following the second feeding, the kestrel was observed to regurgitate and food material exited the nares raising concern for aspiration. Subsequently, he was started on enrofloxacin (Baytril ${ }^{\circledR}$, Bayer Health Care LLC, Shawnee Mission, $\mathrm{KS}, \mathrm{USA}$ ) at $10 \mathrm{mg} / \mathrm{kg}$ intravenously every 12 hours.

The intravenous catheter was removed 72 hours after placement when the kestrel was assessed to be fully hydrated and had become alert enough to disrupt the catheter site. The enrofloxacin and meloxicam were continued via oral administration. Mild paresis of the right leg, likely due to inflammation associated with catheterization, was noted following catheter removal. The patient regained normal use of the leg and foot after several days and continued meloxicam therapy. Gavage feeding was discontinued when the patient readily consumed frozen, thawed adult mice offered on day four. By day five, the OS was determined to be visual again based on an ophthalmic exam. On day seven, the kestrel was observed to be fully flighted and considered to be completely recovered. After completion of a 10-day course of enrofloxacin, the bird was transferred to a raptor rehabilitation facility for reconditioning prior to release.

\section{DISCUSSION AND CONCLUSIONS}

Consideration of the interrelated mechanisms involved in secondary brain injury is useful in guiding the treatment selection. A fixed cranial vault dictates that if one of the components of ICP (blood, cerebral spinal fluid, and brain tissue) increases then the other two must decrease. When this compliance is exhausted, ICP begins to increase, resulting in the observed clinical decline of the patient (Dewey 2000). Signs of increased ICP include bradycardia with concurrent hypertension (Cushing reflex), loss of pupillary light reflex, loss of physiologic nystagmus, altered levels of consciousness, mydriasis, and decerebrate posturing (Syring 2005). Brain herniation is a fatal consequence of increased ICP, and treatment should focus on controlling or preventing ICP increases.

Other key components of neuronal damage include hypoxia and ischemia from decreased cerebral blood flow, hemorrhage, and concurrent respiratory or cardiac injuries (Sande and West 2010). The brain's high metabolic rate causes increased sensitivity to reduced oxygen delivery and subsequent compensation by anaerobic metabolism. This leads to energy shortages resulting in failure of energy-dependant membrane pumps, altered ion concentrations, and ultimately, cellular edema (Dearden 1998). Cerebral edema causes further increases in ICP and neuronal damage from abnormalities in ion homeostasis and free radical production. Hemorrhage can occur from direct trauma, coagulopathies, or hypertension. It leads to ischemia/hypoxia as well as increased ICP. Intracranial hemorrhage also can have a mass effect, leading to ventricular obstruction, impaired drainage of cerebral spinal fluid (CSF), and further increases in ICP. It is important to note that these cases frequently are accompanied by injuries to other body systems, which require management as well.

To prevent irreversible brain damage secondary to the described pathophysiology, TBI treatment requires an immediate, aggressive, multi-modal approach. To preclude the detrimental effects of anaerobic metabo- 
lism in the brain, hypoxia should be avoided at all costs. Stabilization with oxygen therapy must occur prior to any therapies for increased ICP (Syring 2005). Supplemental oxygenation can be provided via flowby masks, a specialized oxygen cage, or an oxygen concentrator and a sealed enclosure. To ensure adequate tissue perfusion, it is important to provide fluid therapy when a patient presents signs of hypovolemia or dehydration (Sande and West 2010).

Minimizing cerebral metabolic rate through limited stimulation is another important means to preventing anaerobic metabolism within the brain. Patients should be placed in a dark, quiet environment.

Seizure activity must be controlled to prevent associated increased neuronal energy demands and intracranial hypertension (Platt 2005). Analgesics including opioids help alleviate the noxious stimulus of pain. Although there is some risk of respiratory depression and hypotension associated with opioids, they have been shown to be safe and to not negatively affect ICP (Lauer et al 1997). Non-steroidal anti-inflammatory agents can limit inflammatory responses that worsen CNS signs as well as provide some level of analgesia. Corticosteroid use is not currently recommended in TBI patients, and in humans has been associated with increased mortality (Sande and West 2010).

Additional supportive care to consider in management of TBI cases includes treatment of shock and dehydration as necessary, providing nutritional support, frequently turning recumbent animals to avoid pressure sore formation, and maintaining clean, dry bedding. For long term cases, physical therapy to limit muscle atrophy as well as expression of the urinary bladder in mammals or placing an indwelling urinary catheter may be required. These prolonged care techniques are controversial in wildlife patients. If noticeable improvement does not occur within 48 to 72 hours, prognosis for the patient to recover enough cognitive function to be released declines significantly. In such cases, humane euthanasia should be considered.

The largest component of TBI treatment revolves around controlling and decreasing ICP. Elevating the head to an angle of 15 to $30^{\circ}$ facilitates fluid drainage to the rest of the body (Dewey 2000). Additionally, jugular compression, coughing, sneezing, and straining should be avoided. Hyperthermia should be prevented because it causes vasodilatation of intracranial vessels, which contributes to ICP elevation (Sande and West 2010). In human and occasionally companion animal medicine, surgical intervention to treat hemorrhage and decrease ICP is possible. However, this is an uncommon and often impractical approach for wildlife cases.
Hyperosmotic agents are a cornerstone of the therapy for decreasing intracranial pressure. Both mannitol and hypertonic saline decrease ICP not only through their osmotic effects, but also by reducing blood viscosity (Ogden et al 2005). Hypertonic saline alone has a transient impact, but when combined with an artificial colloid such as hetastarch, the intravascular osmotic gradient is maintained for several hours (Qureshi and Suarez 2000). There is also the effect of retaining fluids in the intravascular compartment, resulting in volume expansion, which is beneficial in hypovolemic patients. This quality of hypertonic saline with hetastarch makes it a superior choice compared to mannitol in hypovolemic patients given the ability of mannitol to exacerbate a hypovolemic state (Bullock et al 2000).

Mannitol and hypertonic saline have been shown to be equally effective in reducing ICP when administered at the same osmotic burden (Sakellaridis et al 2010). In human patients, when hypertonic saline is administered at higher osmolar loads than mannitol, the hypertonic saline is shown to have a more significant impact on decreasing the number of increased ICP events and lowering mortality (Harutjunyan et al 2005; Wakai et al 2007). At the University of Illinois Wildlife Medical Clinic, the commonly used dosage of 20 percent mannitol is 0.5 to $1.0 \mathrm{~g} / \mathrm{kg}$ infused intravenously over 20 minutes. As described in the case of this kestrel, administration of hypertonic saline and hetastarch results in a 1.5 to 3 times greater osmotic burden than that achieved with mannitol. At these dosages, hypertonic saline with hetastarch may have advantages compared to mannitol in the treatment of increased ICP.

This case illustrates the effectiveness of hypertonic saline with hetastarch as part of a treatment regime for suspected increased ICP in a wildlife patient. The protocol was indicated for this patient instead of mannitol given his hypovolemic state at the time of treatment. Hypertonic saline may be a useful tool in wildlife medicine as it is inexpensive, readily available, and emerging as an alternative to mannitol in other fields of medicine for treatment of increased ICP.

\section{LITERATURE CITED}

Bullock, R. M., R. M. Chestnut, G. L. Clifton, J. Ghajar, D. W. Marion, and R. K. Naryan. 2000. Use of Mannitol. Journal of Neurotrauma. 17: 521-525.

Deardon, N. M. 1998. Mechanisms and Prevention of Secondary Brain Damage during Intensive Care. Clinical Neuropathology. 17(4): 221-228. 
Dewey, C. W. 2000. Emergency Management of the Head Trauma Patient. Veterinary Clinics of North America Small Animal Practice. 30: 207-225.

Harutjunyan, L., C. Holz, A. Rieger, M. Menzel, S. Grond, and J. Soukup. 2005. Efficiency of 7.2\% Hypertonic Saline Hydroxyethyl Starch 200/0.5 versus Mannitol 15\% in the Treatment of Increased Intracranial Pressure in Neurosurgical Patients-A Randomized Clinical Trial. Critical Care. 9(5): R530-R540.

Lauer, K. K., L. A. Connolly, and W. T. Schmeling. 1997. Opioid Sedation Does Not Alter Intracranial Pressure in Head Injured Patients. Canadian Journal of Anesthesia. 44(9): 929-933.

Ogden, A. T., S. A. Mayer, and S. E. Connolly, Jr. 2005. Hyperosmolar Agents in Neurosurgical Practice: The Evolving Role of Hypertonic Saline. Journal of Neurosurgery. 57(2): 207-215.

Platt, S. 2005. Evaluation and Treatment of the Head Trauma Patient. In Practice. 27: 31-35.

Qureshi, A. I., and J. I. Suarez. 2000. Use of Hypertonic Saline Solutions in Treatment of Cerebral Edema and Intracranial Hypertension. Critical Care Medicine. 28(9): 3301-3313.

Sakellaridis, N., E. Pavlou, S. Karatzas, D. Chronis, K. Vlachos, K. Chatzopoulos, E. Dimopoulous, C. Kelesis, and V. Karaouli. 2010. Comparison of Mannitol and Hypertonic Saline in the Treatment of Severe Brain Injuries. Journal of Neurosurgery. [cited 2010 December 4]. Available from: <http://thejns.org/doi/abs/10.3171/2010 .5.JNS091685? prevSearch=allfield\%253A\%2528 Sakellaridis\%2529\&searchHistoryKey=>.

Sande, A., and C. West. 2010. Traumatic Brain Injury: A Review of Pathophysiology and Management. Journal of Veterinary Emergency and Critical Care. 20(2): 177-190.

Syring, R. S. 2005. Assessment and Treatment of Central Nervous System Abnormalities in the Emergency Patient. Veterinary Clinics of North America Small Animal Practice. 35: 343-358.

Wakai, A., I. Roberts, and G. Schierhout. 2007. Mannitol for Acute Traumatic Brain Injury. Cochrane Database Systematic Reviews. (1): CD001049. 正品

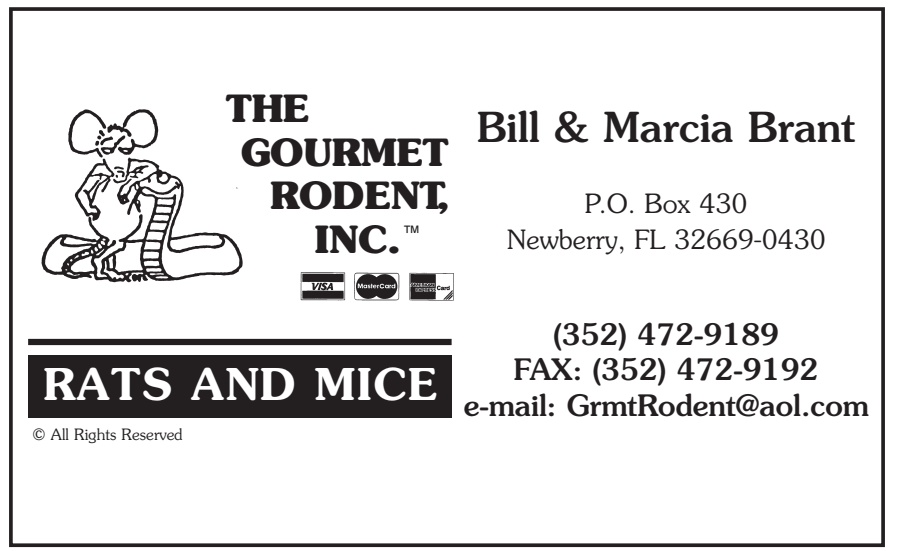

\begin{tabular}{|c|c|}
\hline \multicolumn{2}{|c|}{$\begin{array}{l}\text { Fairfield, } \mathrm{OH} 45014 \\
\text { www.grubco.com }\end{array}$} \\
\hline \multicolumn{2}{|c|}{$\begin{array}{l}\text { Phone orders please call 800-222-3563. } \\
\text { We accept Visa, MasterCard, and Discover. }\end{array}$} \\
\hline \multicolumn{2}{|c|}{ Wildlife rehabilitators receive a $5 \%$ discount! } \\
\hline $\begin{array}{l}\text { Mealworms - Mini, } \\
\text { Small,Medium, or Large. }\end{array}$ & $\begin{array}{l}\text { Waxworms - Rich in } \\
\text { protein, moisture, and } \\
\text { fat. }\end{array}$ \\
\hline \multicolumn{2}{|l|}{ Mighty Mealys ${ }^{\circledR}-$ Twice } \\
\hline $\begin{array}{l}\text { the size of our large } \\
\text { mealworms. These } \\
\text { oversized mealworms } \\
\text { are perfect for larger }\end{array}$ & $\begin{array}{l}\text { Superworms - These fast } \\
\text { moving larvae are great } \\
\text { for feeding reptiles. }\end{array}$ \\
\hline animals. & $\begin{array}{l}\text { Fly Larvae - These larvae } \\
\text { are raised in a vitamin }\end{array}$ \\
\hline $\begin{array}{l}\text { Crickets }-P H, 1 / 4^{\prime \prime}, 1 / 2 " \text {, } \\
3 / 4 " \text {, and 1". }\end{array}$ & and protein rich mixture. \\
\hline
\end{tabular}

